\title{
Erratum: Complete positivity, finite-temperature effects, and additivity of noise for time-local qubit dynamics [Phys. Rev. A 93, 052103 (2016)]
}

Juho Lankinen, Henri Lyyra, Boris Sokolov, Jose Teittinen, Babak Ziaei, and Sabrina Maniscalco

(Received 15 November 2016; published 29 November 2016)

DOI: 10.1103/PhysRevA.94.059904

The parameter $t_{3}(t)$ in Eq. (20) of the main article should be replaced with $\tilde{t}_{3}(t)$, defined as

$$
\tilde{t}_{3}(t):=-e^{-\Gamma(t)}[1+2 G(t)]+1=-t_{3}(t) .
$$

With this change to Eqs. (27) and (28) of the main article, the corrected versions of $p(t)$ and $q(t)$ become

$$
\begin{aligned}
& \tilde{p}(t)=\frac{1}{2}\left[-t_{3}(t)+\lambda_{3}(t)\right]=-q(t), \\
& \tilde{q}(t)=\frac{1}{2}\left[-t_{3}(t)-\lambda_{3}(t)\right]=-p(t),
\end{aligned}
$$

respectively. By applying these corrections to the complete positivity criteria in Eqs. (25) and (26) of the main article one gets

$$
\begin{aligned}
& \tilde{y}(t)^{2} \leqslant\left[\frac{1}{2}-\tilde{p}(t)\right]\left[\frac{1}{2}+\tilde{q}(t)\right]=\left[\frac{1}{2}+q(t)\right]\left[\frac{1}{2}-p(t)\right], \\
& \tilde{w}(t)^{2} \leqslant\left[\frac{1}{2}-\tilde{q}(t)\right]\left[\frac{1}{2}+\tilde{p}(t)\right]=\left[\frac{1}{2}+p(t)\right]\left[\frac{1}{2}-q(t)\right] .
\end{aligned}
$$

Since $\tilde{y}(t)$ and $\tilde{w}(t)$ do not depend on $\tilde{t}_{3}(t)$, they are not affected by the correction. By comparing Eqs. (4) and (5) above with the conditions in Eqs. (25) and (26) of the main article, respectively, we see that the corrected and original conditions are equivalent. Thus, we conclude that the correction in $t_{3}(t)$ has no effect on the complete positivity criteria studied. 